\title{
A LIL FOR OCCUPATION TIMES OF STABLE PROCESSES
}

\author{
NARN-RUEIH SHIEH
}

\begin{abstract}
We prove a Strassen-type law of iterated logarithms for the occupation times of an $R^{d}$-valued $(d \geq 1)$ stable process with the scaling property and positive density functions. An immediate application of our result is to obtain the asymptotic behavior of the occupation times of a path occupied in large spheres.
\end{abstract}

1. Introduction and main result. The purpose of this paper is to prove a Strassen-type law of iterated logarithms for the occupation times of a stable process. First, we review the result in Donsker and Varadhan [3] which is our motivation for study. Let $\{x(s), 0 \leq s<\infty\}$ be Brownian motion in $R^{d}, d \geq 3$ (so that the paths are transient), and let $\sigma(\omega, A)$ denote the total occupation time of a particular path $x(s, \omega)$ occupied in a Borel set $A \subset R^{d}$, i.e.,

$$
\sigma(\omega, A)=\int_{0}^{\infty} \chi_{A}(x(s, \omega)) d s .
$$

For each $\omega, \sigma(\omega, \cdot)$ is a $\sigma$-finite measure on $R^{d}$. If we let $M$ be the space of all $\sigma$-finite measures on $R^{d}$ and impose the vague topology on $M$, then we have

Donsker and Varadhan [3, Theorem 1.1]. Define, for $t \geq 3$,

$$
W_{t}(\omega, A)=\frac{\sigma(\omega, t A)}{t^{2} \log \log t} .
$$

Let $\Phi(\sigma)$ be continuous (in the vague topology) at the points of

$$
\{\sigma \in M: I(\sigma) \leq 1\} \text {. }
$$

Then, for almost all $\omega$,

$$
\varlimsup_{t \rightarrow \infty}\left\{\Phi\left(W_{t}(\omega, \cdot)\right)=\sup _{\sigma: I(\sigma) \leq 1} \Phi(\sigma) .\right.
$$

In the above, $I(\sigma)$, the $I$-function of $\sigma$, is defined by

$$
I(\sigma)=-\inf _{u \in U} \int_{R^{d}}\left(\frac{\frac{1}{2} \Delta u}{u}\right)(x) \sigma(d x),
$$

where $U$ is a suitable subspace of the domain of $\Delta$.

We prove a result analogous to the above theorem of Donsker and Varadhan for general stable processes, including planar Brownian motion (recall that the

Received by the editors October 23, 1985 and, in revised form, March 4, 1986.

1980 Mathematics Subject Classification (1985 Revision). Primary 60F17, 60J30; Secondary 60F10.

Key words and phrases. Stable processes, occupation times, law of iterated logarithms, Ifunctions.

This work is supported in part by National Science Council, Taipei, Taiwan. 
latter is recurrent). We cannot obtain such a result by routine modifications of the procedure in [3], since some specific properties of transient Brownian paths play an indispensable role in that paper. We shall, instead of total occupation times, consider truncated occupation times:

$$
\sigma(\omega, A, t)=\int_{0}^{t} \chi_{A}(x(s, \omega)) d s .
$$

For a stable process $\{x(s), 0 \leq s<\infty\}$ in $R^{d}(d \geq 1)$ with index $\alpha$, we define

$$
Z_{t}(\omega, A)=\frac{\sigma\left(\omega, t A, t^{\alpha} \log \log t\right)}{t^{\alpha} \log \log t}, \quad t \geq 3 .
$$

Now, for each $\omega, Z_{t}(\omega, \cdot)$ is a probability measure (p.m.) on $R^{d}$. Let $M$ now denote the space of all sub-p.m. on $R^{d}$, equipped with the vague topology too. For $\sigma \in M$, define

$$
I(\sigma)=-\inf _{u \in D^{+}} \int_{R^{d}}\left(\frac{L u}{u}\right)(x) \sigma(d x)
$$

where $L$ is the infinitesimal generator of $\{x(s)\}$ and $D^{+}$is the space of positive functions in the domain of $L$ (this definition follows the general definition of the $I$-function described in Donsker and Varadhan [1]). Our result is

THEOREM 1.1. Let $\{x(s), 0 \leq s<\infty\}$ be an $R^{d}$-valued stable process with index $\alpha$. Assume that $\{x(s)\}$ has the scaling property and that $x(s)$ has an everywhere positive $(>0)$ density. Let $\Phi(\sigma)$ be continuous at the points of $B=\{\sigma \in M: I(\sigma) \leq$ 1\}. Then, for almost all $\omega$,

$$
\varlimsup_{t \rightarrow \infty} \Phi\left(Z_{t}(\omega, \cdot)\right)=\sup _{\sigma: I(\sigma) \leq 1} \Phi(\sigma) .
$$

Note that our assumptions on $\{x(s)\}$ exclude nonsymmetric Cauchy processes and subordinators. However, we do not assume the transience of paths. An immediate application of Theorem 1.1, similar to that in [3], is to let $\Phi(\sigma)=\sigma\left(S_{d}(0,1)\right)$, where $s_{d}(0,1)$ is the unit sphere of $R_{d}$, and thus to obtain the asymptotic behavior of the occupation times of the path occupied in large spheres (the case of small spheres [3, Theorem 1.2] is not considered in this paper). Reword it to indicate the asymptotic nature of the result, for large $\lambda$, Donsker and Varadhan $[\mathbf{3}]$ showed that the total occupation time of a transient Brownian path occupied in the sphere $S(0, \lambda)$ is a.s. (const.) $\left(\lambda^{2} \log \log \lambda\right)$; while we show that the same effect happens when we consider the occupation time only up to $\lambda^{2} \log \log \lambda$.

The proof of Theorem 1.1 follows the usual scheme. Let $C_{\omega}$ denote the set of limit points of $\left\{Z_{t}(\omega, \cdot)\right\}$ (as $\left.t \rightarrow \infty\right)$ in $M$. We prove that $B=C_{\omega}$ a.s. In $\S 2$, we list some preliminaries and prove that $B \supset C_{\omega}$, then we prove that $B \subset C_{\omega}$ in $\S 3$.

The author is indebted to the referee for noticing that we should exclude the subordinators in order to apply the estimates of Donsker and Varadhan [2].

2. $C_{\omega} \subset B$ a.s. Let $\{x(s), 0 \leq s<\infty\}$ be an $R^{d}$-valued $(d \geq 1)$ stable process with index $\alpha$. We refer to Taylor [4] for the definition and basic properties of such processes, we assume that $\{x(s)\}$ has the scaling property: $x(s)$ and $r^{-1 / \alpha} x(r s)$ have the same distribution (abbrev. $x(s) \sim r^{-1 / \alpha} x(r s)$ ) for any $r>0$. We also assume that $x(s)$ has an everywhere positive $(>0)$ density. Except nonsymmetric Cauchy processes and subordinators, all other stable processes satisfy our assumptions. 
Recall that $\{x(s)\}$ is transient if and only if $\alpha<d$, but we do not concern ourselves with this aspect in this paper. We may assume that every path $x(\omega, \cdot)$ is right continuous and has left limits everywhere.

Define, for $t \geq 3, \rho_{t}=(t / \log \log t)^{1 / \alpha}$ and

$$
\hat{L}_{t}(\omega, A)=\frac{1}{t} \int_{0}^{t} \chi_{\rho_{t} A}(x(s, \omega)) d s .
$$

The functional $\hat{L}_{t}(\omega, \cdot)$ is the main concern in an important work on Donsker and Varadhan [2], in which "another law of iterated logarithms" was studied.

LEMMA 2.1. For any $t \geq 3$ and any Borel sets $A_{1}, \ldots, A_{r}$ in $R^{d},\left\{Z_{t}\left(\omega, A_{i}\right)\right\}$ has the same joint distribution as that of $\left\{\hat{L}_{t}\left(\omega, A_{i}\right)\right\}$.

PRoOF. Write

$$
\hat{L}_{t}(\omega, A)=\frac{1}{t} \int_{0}^{t} \chi_{t A}\left(\frac{t x(s)}{\rho_{t}}\right) d s
$$

and apply the scaling property $r^{1 / \alpha} x(s) \sim x(r s)$ with $r=\left(\rho_{t} / t\right)^{-\alpha}=t^{\alpha-1}(\log \log t)$.

Thanks to Lemma 2.1, we are allowed to use nice estimates for the distribution of $\hat{L}_{t}(\omega, \cdot)$ appearing in [2]. In fact, our proof of Theorem 1.1 relies on transforming distributional estimates of $Z_{t}(\omega, \cdot)$ to those of $\hat{L}_{t}(\omega, \cdot)$. We should mention that the results in $[2, \S 2]$ can be extended parallel to those $R^{d}$-valued stable processes satisfying our assumptions in Theorem 1.1.

Proposition 2.2 $C_{\omega} \subset$ B. a.s.

To prove this, let $\beta \notin B$ so that $I(\beta)>1$. Since $I(\cdot)$ is lower semicontinuous and $M$ is compact, we may find a weak neighborhood $N$ of $\beta$ such that $\theta=$ $\inf _{\sigma \in \bar{N}} I(\sigma)>1$. We may assume that $N$ is of the form

$$
N=\left\{\sigma \in M:\left|\int \phi_{i}(x) \sigma(d x)-\int \phi_{i}(x) \beta(d x)\right|<\varepsilon, 1 \leq i \leq r\right\},
$$

where $\varepsilon>0$ and $\phi_{i}(x) \in C_{0}^{\infty}\left(R^{d}\right)$.

Choose $k$ such that $0<k<1$ and $k(1+\theta)>2$. Set $t_{n}=\exp \left(n^{k}\right)$ and write $Z_{n}=Z_{t_{n}}, \hat{L}_{n}=\hat{L}_{t_{n}}$. We prove that

$$
P\left(\omega: Z_{n}(\omega, \cdot) \in N \text { i.o. }\right)=0 .
$$

Note that

$$
\begin{aligned}
P\left(\omega: Z_{n}(\omega, \cdot) \in N\right) & =P\left(\omega:\left|\int \phi_{i}(x) Z_{n}(\omega, d x)-\int \phi_{i}(x) \beta(d x)\right|<\varepsilon, 1 \leq i \leq r\right) \\
& =P\left(\omega:\left|\int \phi_{i}(x) \hat{L}_{n}(\omega, d x)-\int \phi_{i}(x) \beta(d x)\right|<\varepsilon, i \leq i \leq r\right) \\
& =P\left(\omega: \hat{L}_{n}(\omega, \cdot) \in N\right) \\
& \leq P\left(\omega: \hat{L}_{n}(\omega, \cdot) \in \bar{N}\right),
\end{aligned}
$$

in the above, the second equality is due to Lemma 2.1. Then, we can use (2.30)(2.32) in [2] to conclude that $\sum P\left(\omega: Z_{n}(\omega, \cdot) \in N\right)<\infty$. Hence, (2.2) is proved, by the Borel-Cantelli Lemma. Let $N_{1}$ be another weak neighborhood of $\beta$ such that $N_{1} \subset \bar{N}_{1} \subset N$. We prove that: for each $\omega$ such that $Z_{n}(\omega, \cdot) \in N$ for only finitely 
many $n, Z_{t}(\omega, \cdot) \notin N_{1}$ when $t$ is sufficiently large. Assume the contrary; then there exists $\tau_{j} \uparrow \infty$ such that $Z_{\tau_{j}}(\omega, \cdot) \in N_{1}$, for all $\tau_{j}$. Let $t_{n_{j}}: t_{n_{j}-1} \leq \tau_{j} \leq t_{n_{j}}$. Since $\left(t_{n-1} / t_{n}\right) \rightarrow 1$ as $n \rightarrow \infty$ (due to our choice that $0<k<1$ ), the above conclusion will contradict the observation that

$$
\left\|Z_{t_{2}}(\omega, \cdot)-Z_{t_{1}}(\omega, \cdot)\right\| \rightarrow 0 \text { as } t_{2}>t_{1} \rightarrow \infty \text { and }\left(t_{1} / t_{2}\right) \rightarrow 1,
$$

where $\|\cdot\|$ denotes the variation norm of measures.

Thus, we have proved that, for almost all $\omega, Z_{t}(\omega, \cdot) \notin N_{1}$ when $t$ is sufficiently large; the latter means that $\beta \notin C_{\omega}$. Therefore, Proposition 2.2 is proved.

3. $\beta \subset C_{\omega}$ a.s. Now, we prove that each sub-p.m. $\beta$ with $I(\beta) \leq 1$ is in $C_{\omega}$. In view of [2, Lemma 2.16], it suffices to prove

THEOREM 3.1. Let $\beta$ be a sub-p.m. on $R^{d}$ such that $I(\beta)<1$ and such that $\beta$ is supported on $|x|<a$ for some $a>0$. Let $G$ be a weak neighborhood of $\beta$; define

$$
E_{t}=\left\{\omega: Z_{t}(\omega, \cdot) \in G\right\}
$$

Then, for almost all $\omega, \omega \in E_{t}$ for a sequence of $t$ 's increasing to $\infty$.

To prove Theorem 3.1, we define an associated p.m. of $Z_{t}(\omega, \cdot)$ by

$$
\begin{array}{r}
\bar{Z}_{t_{1}, t_{2}}(\omega, A)=\frac{1}{\left(t_{2}^{\alpha} \log \log t_{2}\right)\left(1-\left(t_{1} / t_{2}\right)\right)} \int_{\left(t_{2}^{\alpha} \log \log t_{2}\right)\left(t_{1} / t_{2}\right)}^{t_{2}^{\alpha} \log \log t_{2}} \chi_{t_{2} A}(x(s)) d s, \\
0<t_{1}<t_{2} .
\end{array}
$$

LEMMA 3.2. If $t_{1}, t_{2} \rightarrow \infty$ and $\left(t_{1} / t_{2}\right) \rightarrow 0$, then

$$
\left\|\bar{Z}_{t_{1}, t_{2}}(\omega, \cdot)-Z_{t_{2}}(\omega, \cdot)\right\| \rightarrow 0 .
$$

ProOF. Break the integration interval $\left[0, t_{2}^{\alpha} \log \log t_{2}\right]$ of $Z_{t_{2}}(\omega, \cdot)$ into $\left[0,\left(t_{2}^{\alpha} \log \log t_{2}\right)\left(t_{1} / t_{2}\right)\right]$ and $\left[\left(t_{2}^{\alpha} \log \log t_{2}\right)\left(t_{1} / t_{2}\right), t_{2}^{\alpha} \log \log t_{2}\right]$.

Return to the proof of Theorem 3.1. Since $I(\beta)=\theta<1$, we can choose $k>1$ but such that $(2 k-1)(\theta+1)<2$. Set $t_{n}=\exp \left[n^{k}\right]$. Since $k>1,\left(t_{n-1} / t_{n}\right) \rightarrow 0$ as $n \rightarrow \infty$. Let $G_{1}$ be another weak neighborhood of $\beta$ such that $G_{1}$ is of the form (2.1) and such that $G_{1} \subset \bar{G}_{1} \subset G$. Write $r_{n}=t_{n}^{\alpha} \log \log t_{n}, e_{n}=t_{n-1} / t_{n}$, $\theta_{n} \doteq t_{n}^{\alpha-1} \log \log t_{n}, \bar{Z}_{n}=\bar{Z}_{t_{n-1}, t_{n}}$, etc. Fix $a^{\prime}: a<a^{\prime}$; define

$$
E_{n}=\left\{\omega: \bar{Z}_{n}(\omega, \cdot) \in G_{1}, \sup _{0 \leq s \leq r_{n} e_{n}}|x(s)|<a t_{n}, \sup _{0 \leq s \leq r_{n}}|x(s)|<a^{\prime} t_{n}\right\} .
$$

Because of Lemma 3.2, Theorem 3.1 will be proved once we can show that

$$
P\left(E_{n} \text { i.o. }\right)=1 \text {. }
$$

Let

$$
\mathcal{F}_{n}=\sigma\left\{x(s): 0 \leq s \leq r_{n} e_{n}\right\} .
$$

Since $r_{n+1} e_{n+1}>r_{n}, E_{n} \in \mathcal{F}_{n+1}$. Thus, by a conditional Borel-Cantelli Lemma ([2, Lemma 2.14], but it seems to exist in the literature already), (3.1) is implied by

$$
\sum_{n} P\left(E_{n} \mid \mathcal{F}_{n}\right)=\infty \quad \text { a.s. }
$$


By the Markov property, we have

$$
P\left(E_{n} \mid \mathcal{F}_{n}\right)=\chi_{B_{n}}(\omega) P_{x\left(r_{n} e_{n}, \omega\right)}\left\{\omega^{\prime}: \tilde{Z}_{n}\left(\omega^{\prime}, \cdot\right) \in G_{1}, \sup _{0 \leq s \leq r_{n}\left(1-e_{n}\right)}\left|x\left(s, \omega^{\prime}\right)\right|<a^{\prime} t_{n}\right\},
$$

where $B_{n}=\left\{\omega: \sup _{1 \leq s \leq r_{n} e_{n}}|x(s, \omega)|<a t_{n}\right\}, \tilde{Z}_{n}(\omega, A)=$ the integral obtained by replacing $x(s)$ in $\bar{Z}_{n}(\omega, \bar{A})$ by $x\left(s-r_{n} e_{n}\right)$, and $P_{x}$ denotes the p.m. corresponding to the process starting at $x$. Define

$$
\rho_{n}=\left(t_{n} / \log \log t_{n}\right)^{1 / \alpha}, \quad \text { and } \quad L_{n}^{\prime}(\omega, A)=\frac{1}{t_{n}-t_{n-1}} \int_{0}^{t_{n}-t_{n-1}} \chi_{\rho_{n} A}(x(s)) d s .
$$

Applying the scaling property $x(s) \sim \theta_{n}^{-1 / \alpha} x\left(\theta_{n} s\right)$, we see that

$$
\inf _{|x| \leq a \rho_{n}} P_{x}\left\{L_{n}^{\prime}(\omega, \cdot) \in G_{1}, \sup _{0 \leq s \leq t_{n}-t_{n-1}}|x(s)|<a^{\prime} \rho_{n}\right\}
$$

is equal to

$$
\inf _{|x| \leq a t_{n}} P_{x}\left\{\tilde{Z}_{n}(\omega, \cdot) \in G_{1} \sup _{0 \leq s \leq r_{n}\left(1-e_{n}\right)}|x(s)|<a^{\prime} t_{n}\right\} .
$$

Since (3.3) is the one appearing in $[2,(2.50)]$, we can use $[2,(2.50)-(2.53)]$ to obtain that

$$
\sum_{n} P\left(E_{n} \mid \digamma_{n}\right) \geq \sum_{n} \frac{\chi_{B_{n}}(\omega)}{n^{\theta^{\prime}}}, \quad \theta^{\prime}<1 .
$$

We may use again the above scaling property and the arguments below $[2,(2.54)]$ to conclude that the last sum in (3.5) is $\infty$ a.s. Therefore (3.1) is proved.

\section{REFERENCES}

1. M. D. Donsker and S. R. S. Varadhan, Asymptotic evaluation of certain Markov process expectations for large times. III, Comm. Pure Apply. Math. 29 (1976), 389-461.

2. 707-753.

3. $\ldots$, A law of the iterated logarithm for total occupation times of transient Brownian motion, Comm. Pure Appl. Math. 33 (1980), 365-393.

4. S. J. Taylor, Sample path properties of processes with stationary independent increments, Stochastic Analysis, Wiley, London, 1973. CHINA

Department of Mathematics, National Taiwan University, Taipei, Taiwan,

Current address: Department of Mathematics, Louisiana State University, Baton Rouge, Louisiana 70803-4918 